POS $\quad$ PROCEEDINGS

\title{
Multiquarks and exotics in the heavy-hadron spectra
}

\author{
A. Valcarce ${ }^{* \dagger}$ \\ Departamento de Física Fundamental e IUFFyM, Universidad de Salamanca, E-37008 \\ Salamanca, Spain \\ E-mail: valcarce@usal.es
}

\section{J. Vijande}

Departamento de Física Atómica, Molecular y Nuclear, Universidad de Valencia (UV) and IFIC (UV-CSIC), E-46100 Valencia, Spain

E-mail: javier.vijande@uv.es

\section{T.F. Caramés}

Departamento de Física Fundamental e IUFFyM, Universidad de Salamanca, E-37008

Salamanca, Spain

E-mail: carameseusal.es

\begin{abstract}
We review our recent studies to unravel the origin of the $X Y Z$ mesons and other multiquark states, like exotics and dibaryons, in the heavy-hadron spectra. Our discussion is made in the framework of a standard nonrelativistic quark-model picture. We discuss the conditions required for the existence of multiquark bound states or resonances contributing to the heavy hadron spectra, being either compact or molecular. The role played by entangled hadron-hadron thresholds is discussed.
\end{abstract}

VIII International Workshop On Charm Physics

5-9 September, 2016

Bologna, Italy

* Speaker.

$\dagger$ This work has been partially funded by Ministerio de Educación y Ciencia and EU FEDER under Contracts No. FPA2013-47443 and FPA2015-69714-REDT, by Junta de Castilla y León under Contract No. SA041U16, by USALFAPESP grant 2015/50326-5, and by the Programa Propio XIII of the University of Salamanca. 


\section{Introduction}

The question we deal with in this note it is not whether it is possible to design a model, or a formalism, able to match one of the newly observed exotic states in the heavy hadron spectra with a particular set of quantum numbers but to understand where the attraction may come from and to explain the systematics that predicts where, if anywhere, experimentalists and theoreticians alike should look into.

When dealing with higher order Fock space contributions to hadron spectroscopy, one has to discriminate between possible multiquark bound states or resonances and simple pieces of the hadron-hadron continuum. For this purpose, one has to analyze the two-hadron states that constitute the threshold for each set of quantum numbers. These thresholds have to be determined assuming quantum number conservation within exactly the same scheme (parameters and interactions) used for the multiquark calculation. If other models, parametrizations or experimental masses are used, then multiquark states might be misidentified as members of the hadron spectra while being simple pieces of the continuum.

\section{XYZ mesons: $(Q \bar{Q} n \bar{n})$ states.}

Four-quark states containing a heavy quark and its corresponding heavy antiquark $(Q \bar{Q} n \bar{n})$, (in the following $n$ stands for a light quark and $Q$ for a heavy $c$ or $b$ quark), show two different thresholds: namely $(Q \bar{Q})(n \bar{n})$ and $(Q \bar{n})(n \bar{Q})$. It has been proved [1] that ground state solutions of the Schrödinger $\left(q_{1} \bar{q}_{2}\right)$ two-body problem are concave in $\left(m_{q_{1}}^{-1}+m_{q_{2}}^{-1}\right)$ and hence $M_{Q_{\bar{n}}}+M_{\bar{Q}_{n}} \geqslant$ $M_{Q \bar{Q}}+M_{n \bar{n}}$ (see Fig. 1 of Ref. [2]). The interaction between the heavy, $(Q \bar{Q})$, and light, $(n \bar{n})$, mesons forming the lowest threshold is almost negligible due to the absence of a light pseudoscalar exchange mechanism between them [3]. Hence, any attractive effect in the four-quark system must have its origin in the interaction of the higher channel $(Q \bar{n})(n \bar{Q})$ or due to the coupled channel effect of the two thresholds, $(Q \bar{Q})(n \bar{n}) \leftrightarrow(Q \bar{n})(n \bar{Q})$ [4]. This has been found to occur for $Q=c$ for the quantum numbers $J^{P C}=1^{++}$, originating the $\mathrm{X}(3872)$ [5].

Although the increase of the mass of the heavy quark tends to enhance the binding of a stable multiquark, the effect of channel coupling may be minimized owing to a change in the masses of the coupled thresholds. This effect has been tested with the hyperspherical harmonic $(\mathrm{HH})$ formalism within the constituent quark model in Ref. [6], analyzing the isoscalar bottom counterpart of the $X(3872),(b \bar{b} n \bar{n})$ with quantum numbers $J^{P C}=1^{++}$. The corresponding lowest thresholds, $B \bar{B}^{*}(10611 \mathrm{MeV})$ and $\Upsilon \omega(10155 \mathrm{MeV})$, are $456 \mathrm{MeV}$ apart. We show in Fig. 1(upper panel) the convergence pattern of the energy of the four-quark system as a function of the hyperangular momenta $K$. It can be clearly seen how the energy of the four-quark system (red line) is converging to the lowest threshold $\Upsilon \omega$ (horizontal blue line), what is a sharp signal of an unbound state. One could however play around with the model parameters to almost degenerate both thresholds by adding attraction in the heavy-light $b n$ sector by slightly increasing the $\alpha_{s}(b n)$ strong coupling constant, what would also increase the coupled channel effect strengthening the $B \bar{B}^{*} \leftrightarrow \Upsilon \omega$ transition interaction. When this is done (green line) the energy drops below threshold and a bound state emerges. One may wonder if only the close-to-degeneracy of the thresholds is sufficient to bind this type of four-quark systems. If this was the case, then the charged partner of this four- 

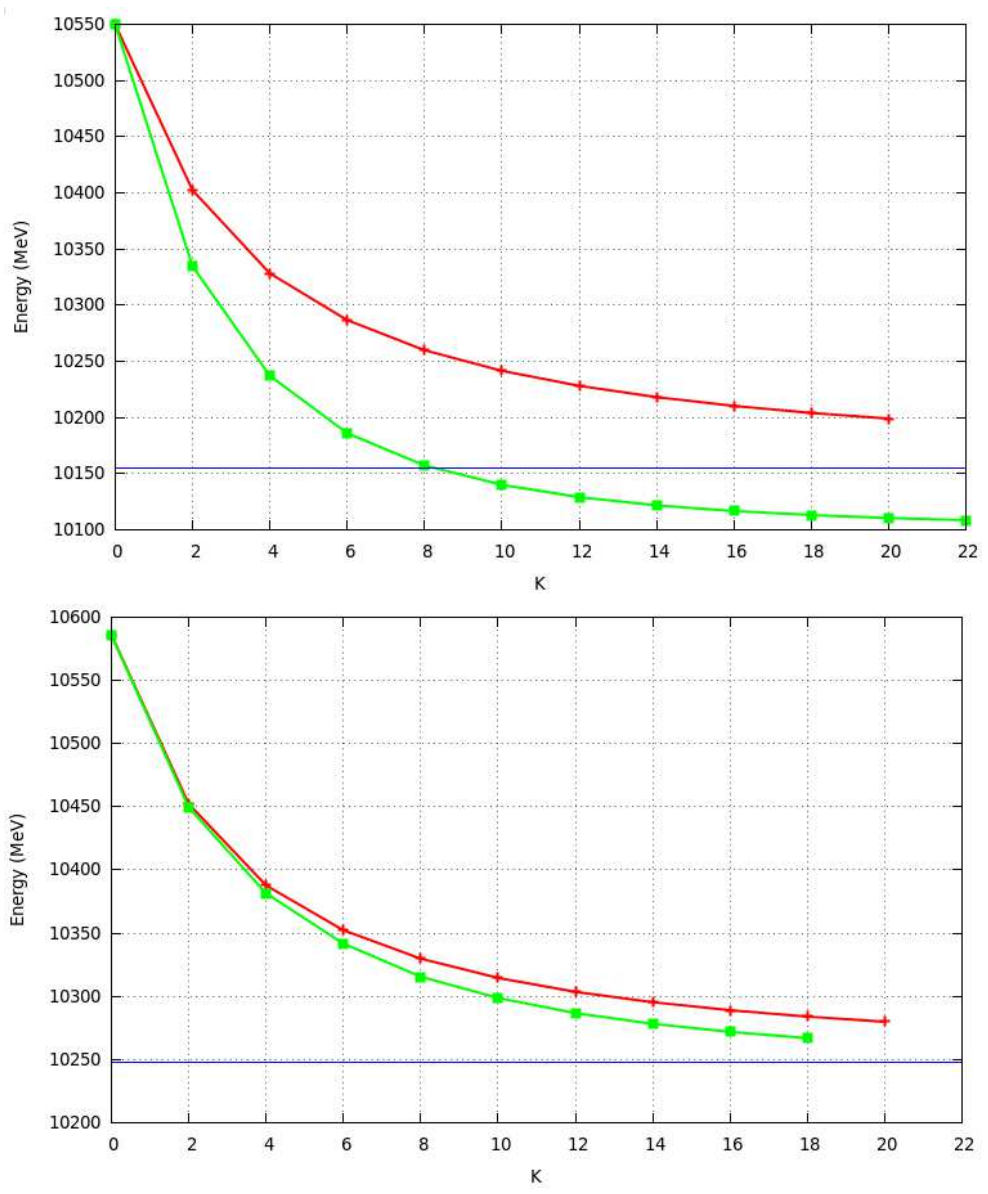

Figure 1: Convergence of $(b \bar{b} n \bar{n})$ with quantum numbers $L=0, S=1, C=+1 P=+1, T=0$ (upper panel) and $T=1$ (lower panel). Red lines correspond to the case where the thresholds are non-degenerate and green lines to the case where they are almost degenerate.

quark state $(T=1)$ should behave exactly in the same manner. However, amazingly this is not so. In Fig. 1(lower panel) we depict the convergence of the isovector state as a function of $K$ for both cases, non-degenerate thresholds (red line) and almost degenerate ones (green line). In this case the lowest threshold would be $\Upsilon \rho(10248 \mathrm{MeV})$. It can be observed that in both cases the four-quark state converges to the lowest threshold and does not form a bound state.

Thus, when the $(Q \bar{Q})(n \bar{n})$ and $(Q \bar{n})(n \bar{Q})$ thresholds are sufficiently far away, no bound states are found for any set of parameters. However, when the thresholds move closer, i.e., the attraction in the higher two-meson state and the coupled channel strength are simultaneously increased, bound states may appear for a subset of quantum numbers. Hence, threshold vicinity is a required but not sufficient condition to bind a four-quark state. An additional condition is required to allow the emergence of such bound states. Such condition is the existence of an attractive interaction in the higher $(Q \bar{n})(n \bar{Q})$ two-meson system that would also give rise to a strong $(Q \bar{Q})(n \bar{n}) \leftrightarrow(Q \bar{n})(n \bar{Q})$ coupling. 


\section{Exotics: $(Q Q \bar{n} \bar{n})$ states.}

If the four-quark system contains identical quarks, like for instance $(Q Q \bar{n} \bar{n})$, the two thresholds are identical, i.e., $(Q \bar{n})(Q \bar{n})$. The importance of this particular feature lies on the fact that a modification of the four-quark interaction would not necessarily translate into the mass of the two free-meson state. Therefore, the unique necessary condition required to have a four-quark bound state would be the existence of a sufficiently attractive interaction between quarks that do not coexist in the two free-meson states. This hypothesis was demonstrated by means of the Lippmann-Schwinger formalism in Ref. [7], concluding the existence of a single stable isoscalar doubly charmed meson with quantum numbers $J^{P}=1^{+}$. This calculation confirmed earlier results by Janc and Rosina [8] predicting the stability of $(c c \bar{u} \bar{d})$ using a quark model fitting ordinary hadrons and by Vijande et al. [9] in a HH approach.

In the case of isoscalar $(Q Q \bar{n} \bar{n})$ states with spin-parity $J^{P}=1^{+}$, there is the fortunate cooperation of two effects. First, the chromoelectric interaction (CE), even if alone, gives stability below the $(Q \bar{n})+(Q \bar{n})$ threshold if the quark-to-antiquark mass ratio is large enough, as it takes advantage of the deeper binding of the $Q Q$ pair $[10,11]$. Second, the chromomagnetic interaction (CM) between the light quarks is also favorable [12]. This configuration is also pointed out as a good candidate for a stable exotic in other approaches such as effective lagrangians [13], lattice QCD [14] or QCD sum rules [15].

\section{Multiquark heavy baryons: $(Q n n n \bar{n})$ states.}

Similar arguments could be used in the case of the heavy baryon spectra. Given a general five-quark state contributing to the heavy baryon spectrum, $(Q n n n \bar{n})$, two different thresholds are allowed, $(n n n)(Q \bar{n})$ and $(Q n n)(n \bar{n})$. A straightforward generalization of the concave behavior in $\left(m_{q_{1}}^{-1}+m_{q_{2}}^{-1}\right)$ of the ground state solutions of the Schrödinger $\left(q_{1} \bar{q}_{2}\right)$ two-body problem to the five-quark system could be obtained within a quark-diquark model if $m_{q_{1}} \leq m_{q_{2}} \leq m_{q_{3}}$. Then $M_{q_{3} \bar{q}_{2}}+M_{q_{1} \bar{q}_{1}} \leq M_{q_{3} \bar{q}_{1}}+M_{q_{1} \bar{q}_{2}}$, because the intervals in $1 / \mu$ of the left and right hand sides have the same middle, but the left interval is wider that the right one. Now, in a crude quark-diquark model, one can translate this as $M_{q_{3} q_{1} q_{1}}+M_{q_{1} \bar{q}_{1}} \leq M_{q_{3} \bar{q}_{1}}+M_{q_{1} q_{1} q_{1}}$, as it is observed in Fig. 5 of Ref. [16], except for the higher spin states where the angular momentum coupling rules impose further restrictions.

An important source of attraction might be the coupled-channel effect of the two thresholds, $(n n n)(Q \bar{n}) \leftrightarrow(Q n n)(n \bar{n})$. The efficiency of this mechanism was tested by a coupled channel calculation considering all physical channels, $(n n n)(Q \bar{n})$ and $(Q n n)(n \bar{n})$. When the $(n n n)(Q \bar{n})$ and $(Q n n)(n \bar{n})$ thresholds are sufficiently far away, the coupled-channel effect is small, and bound states are not found. However, when the thresholds move closer, the coupled-channel strength is increased, and bound states may appear for a subset of quantum numbers. Under these conditions, there are the channels with high spin $J^{P}=5 / 2^{-}$the only ones that may lodge a compact five-quark state for all isospins [16]. The reason stems on the reverse of the ordering of the thresholds, being the lowest threshold $(n n n)(Q \bar{n})$ the one with the more attractive interaction. Of particular interest is the $(T) J^{P}=(2) 5 / 2^{-}$state, that survives the consideration of the break apart thresholds. It may correspond to the $\Theta_{c}(3250)$ pentaquark found by the QCD sum rule analysis of Ref. [17] when 
studying the unexplained structure with a mass of $3250 \mathrm{MeV} / \mathrm{c}^{2}$ in the $\Sigma_{c}^{++} \pi^{-} \pi^{-}$invariant mass reported recently by the BABAR Collaboration [18]. Such state could therefore be a consequence of the close-to-degeneracy of the lowest thresholds with $T=2$ and $J^{P}=5 / 2^{-}, \Delta D^{*}$ and $\Sigma_{c}^{*} \rho$ and the attractive interaction of the $\Delta D^{*}$ system [16].

\section{Exotic pentaquarks: ( $\bar{Q} n n n n)$ states.}

We have studied the ( $\bar{c} n n n n)$ system in Ref. [19]. The most attractive states were the $(T, J)=$ $(2,3 / 2)$ and $(T, J)=(1,5 / 2)$. The state with quantum numbers $(T) J^{P}=(1) 5 / 2^{-}$shows a bound state with a binding energy of $3.9 \mathrm{MeV}$. It corresponds to a unique physical system, $\Delta \bar{D}^{*}$. The $(T, J)=(2,3 / 2)$ state contains a coupled-channel problem, $\Delta \bar{D}-\Delta \bar{D}^{*}$. While the diagonal interactions are not even attractive, the coupling between them is strong because the decay $\bar{D}^{*} \rightarrow \bar{D}+\pi$ is allowed, but not enough to generate a bound state.

When the mass of the heavy meson is increased the contribution of the kinetic energy is reduced. Thus, the binding energy of the ( $\bar{b} n n n n)$ system is expected to be slightly larger than in the ( $\bar{c} n n n$ ) case, because $\bar{D}$ and $B$ mesons have similar interactions with nucleons due to having the same quark structure. Thus, we repeated the calculation for the ( $\bar{b} n n n n)$ system looking for deeper bound states in a baryon-meson system with a heavier antiquark. Surprisingly, the increment in the attraction is not regularly spread over the different $(T, J)$ channels. The $(T, J)=(2,3 / 2)$ is strongly affected and becomes the lowest one with an important gain of binding, showing a bound state with a binding energy of $42 \mathrm{MeV}$. The ordering of the attractive channels is therefore reversed with respect to the (innnn) case.

Which is the responsible for this unexpected behavior of the binding energy as the mass of the heavy meson augments? The reason lies on the internal structure of the states and the behavior of the thresholds when increasing the heavy meson mass. As mentioned above, the $(T, J)=(1,5 / 2)$ state is made of a unique physical system, $\Delta \bar{D}^{*}$ in the charm sector or $\Delta B^{*}$ in the bottom sector, and thus there are no coupled-channel effects. Moving from the charm to the bottom sector gives rise to a small gain of binding, from $3.9 \mathrm{MeV}$ in the $N \bar{D}$ system to $5.3 \mathrm{MeV}$ in the $N B$ system, as one would naively have expected due to a smaller kinetic energy contribution but keeping a rather similar interaction. However, the $(T, J)=(2,3 / 2)$ state contains a coupled-channel problem, $\Delta \bar{D}-\Delta \bar{D}^{*}$ in the charm sector and $\Delta B-\Delta B^{*}$ in the bottom sector. Whereas the diagonal potentials are not strong, as mentioned above, the coupling between the two channels is important because the $\bar{D}^{*} \rightarrow \bar{D}+\pi$ or $B^{*} \rightarrow B+\pi$ decays. When moving from the charm to the bottom sector the most important effect is the reduction of the mass difference between the two thresholds contributing to this state. The mass difference between vector and pseudoscalar mesons scales as predicted by the chromomagnetic interaction, $1 / m_{q} m_{Q}$ [20]. This means a reduction around a factor 3 when going from open-charm to open-bottom mesons. In particular, while $M\left(\Delta \bar{D}^{*}\right)-M(\Delta \bar{D})=141 \mathrm{MeV}$, $M\left(\Delta B^{*}\right)-M(\Delta B)=45 \mathrm{MeV}$ what makes the coupled-channel effect much more important in the bottom sector and reverses the order of the two attractive channels, making the $(T, J)=(2,3 / 2)$ state the lowest one. Thus, when going from the charm to the bottom sector in the baryon-meson open-flavor region, the number of states and their ordering may be modified due to the presence of nearby thresholds. Such effect seems difficult to be predicted by any systematic expansion of the heavy quark sector. 


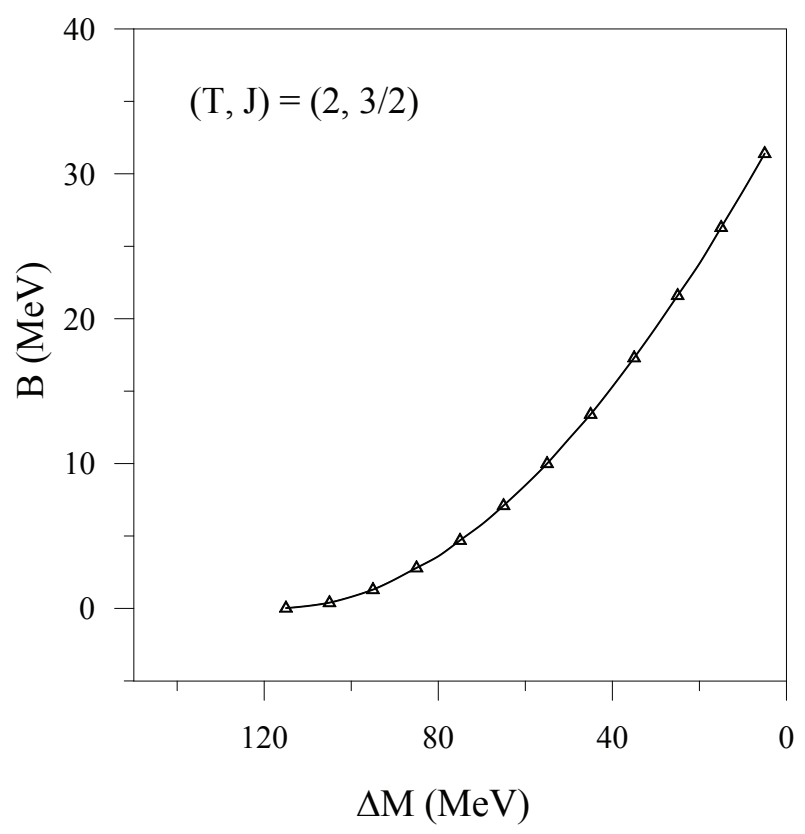

Figure 2: $(T, J)=(2,3 / 2) \bar{c} n n n n$ binding energy, B in $\mathrm{MeV}$, as a function of $\Delta M=M\left(\Delta \bar{D}^{*}\right)-M(\Delta \bar{D})$ mass difference. Note that for the experimental value, $141 \mathrm{MeV}$, the system is not bound.

To illustrate our results, we have plotted in Fig. 2 the evolution of the binding energy of the $(T, J)=(2,3 / 2)(\bar{c} n n n n)$ state if we artificially diminish the mass difference between the vector $\bar{D}^{*}$ and the pseudoscalar $\bar{D}$ mesons. We can see how a bound state arises when the thresholds come closer, around $120 \mathrm{MeV}$, without modifying the interactions entering the problem. Besides, the binding energy increases when the mass difference is reduced. This result poses an important warning when trying to extrapolate results of binding energies of two-hadron systems to different flavor sectors. If the binding is mainly due to the vicinity of coupled thresholds, it may be diminished by the increase of the mass of the two-hadron system if it separates the thresholds [6]. Thus, if this mechanism is working for some of the recently discovered pentaquark states at the LHCb or the exotic states discovered in the hidden-charm or hidden-beauty meson spectra, the pattern expected on different flavor sectors may differ significantly as opposite to the charmonium and bottomonium spectra or the charm and bottom baryon spectra below open-flavor thresholds.

\section{Dibaryons: (QQnnnn) states.}

We finally show results about the stability of hexaquark systems containing two heavy quarks and four light quarks within a simple quark model containing only confinement, chromoelectric and chromomagnetic effects, but considering the full color-spin basis contributing to the six-quark problem. No bound or metastable state is found [21].

The scalar state $J^{P}=0^{+}$with isospin $T=1 / 2,3 / 2$, that would be degenerate because the potential does not depend on the total isospin, would stand, for example, for a flavor content (uudscc). In this case thirteen different color-spin vectors are allowed by antisymmetry requirements. To construct the basis of color and spin states, we formally consider the system as a set of three two-quark 
subsystems, $(q q)(q q)\left(Q Q^{\prime}\right)$, with color $\overline{3}$ or 6 and spin 0 or 1 . We built the most general basis compatible with an overall color singlet and spin 0 state. The requirements of antisymmetrization are strictly enforced for all states which are shown. The relevant vectors in color space are: $C_{1}=(666), C_{2}=(6 \overline{3} \overline{3}), C_{3}=(\overline{3} 6 \overline{3}), C_{4}=(\overline{3} \overline{3} 6)$; and in spin space: $S_{1}=(000), S_{2}=(011)$, $S_{3}=(101), S_{4}=(110)$. The two thresholds allowed for the dissociation of the $J^{P}=0^{+}$six-quark state would have energies: $q q Q+q q Q^{\prime}=2.630 \mathrm{GeV}$ and $Q Q^{\prime} q+q q q=2.570 \mathrm{GeV}$. We give in Table 1 the probabilities of the channels contributing to the coupled channel calculation. The final

Table 1: Probabilities of the different six-body channels contributing to the $J^{P}=0^{+}$six-quark state.

\begin{tabular}{ccccc} 
Channel & $C_{1} S_{2}$ & $C_{2} S_{1}$ & $C_{3} S_{4}$ & $C_{4} S_{3}$ \\
\hline Probability & 0.004 & 0.539 & 0.456 & 0.001
\end{tabular}

result we have obtained is $2.767 \mathrm{GeV}$. This is the sign of either the absence of a bound state, or, at most, of a very tiny binding. This is confirmed by the use of the alternative bases, what means that neither the residual color-singlet exchange between the two clusters, nor the coupling of the different baryon-baryon thresholds is sufficient to bind the system.

We have checked that in the infinite mass limit for the mass of the heavy quarks the system gets binding with respect to the upper threshold, $(q q Q)+\left(q q Q^{\prime}\right)$, but it is always above the lowest one $\left(Q Q^{\prime} q\right)+(q q q)$. For example, for $M=10 \mathrm{GeV}$ and $m=0.4 \mathrm{GeV}$ we get $2.326 \mathrm{GeV}$ for the energy of the six-quark state in the coupled channel calculation, while the thresholds come given by $E\left(Q Q^{\prime} q\right)+E(q q q)=2.162 \mathrm{GeV}$ and $E(q q Q)+E\left(q q Q^{\prime}\right)=2.477 \mathrm{GeV}$. The six-quark state, that it is now in between the two thresholds, is described by the same color-spin vectors shown in Table $1, C_{2} S_{1}$ and $C_{3} S_{4}$, where the two-heavy quarks are in a $\overline{3}$ color state, that would split into the lowest threshold. In other words, the two-heavy quarks control the mass of the six-body state in the infinite mass limit.

We now try to explain why these results are plausible. For the CM part, the subject is already well documented with the discussions around the $H$ dibaryon [22]. The effects of $\mathrm{SU}(3)_{\mathrm{F}}$ breaking, a different mass for the strange quark, tends to spoil the promises of binding based on the sole spincolor algebra, and, more important, the short-range correlation factors are significantly smaller in a multiquark than in baryons. As for the CE part, a superficial analysis would argue that, as soon as $-\sum \tilde{\lambda}_{i} \cdot \tilde{\lambda}_{j}$ is locked to 16 in any spin-color channel $\left|C_{a}\right\rangle\left|S_{b}\right\rangle$, the CE part of the binding will remain basically untouched, independent of the combination of the $\left|C_{a}\right\rangle\left|S_{b}\right\rangle$ dictated by the CM part. However, this is not the case. For equal masses, the deepest CE binding is obtained when the distribution of CE strength factors $\left\{-\tilde{\lambda}_{i} \cdot \tilde{\lambda}_{j}\right\}$ is the most asymmetric [23], which favors the threshold against a compact multiquark. For a mass distribution such as $\left(q q q q Q Q^{\prime}\right), \mathrm{CE}$ dynamics favors the $Q Q^{\prime}$ two-quark state being in a color $\overline{3}$ state. Once this is enforced, the best $\mathrm{CE}$ energy is obtained when the $Q q$ and $Q^{\prime} q$ pairs receive the largest strength, and they come with a larger reduced mass than $q q$. This can be checked explicitly in a simple solvable model with an interaction proportional to $-\tilde{\lambda}_{i} \cdot \tilde{\lambda}_{j} r_{i j}^{2}$. However, CM effects are optimized when the light sector receives the largest color strengths. Hence, there is somewhat a conflict between CE and CM effects, and this explains the lack of bound states in our model. 


\section{References}

[1] R. A. Bertlmann and A. Martin, Nucl. Phys. B 168, 111 (1980).

[2] T. F. Caramés, A. Valcarce, and J. Vijande, Phys. Lett. B 709, 358 (2012).

[3] N. A. Törnqvist, Z. Phys. C 61, 525 (1994).

[4] M. F. M. Lutz and E. E. Kolomeitsev, Nucl. Phys. A 755, 29c (2005).

[5] T. Fernández-Caramés, A. Valcarce, and J. Vijande, Phys. Rev. Lett. 103, 222001 (2009).

[6] J. Vijande and A. Valcarce, Phys. Lett. B 736, 325 (2014).

[7] T. F. Caramés, A. Valcarce, and J. Vijande, Phys. Lett. B 699, 291 (2011).

[8] D. Janc and M. Rosina, Few Body Syst. 35, 175 (2004).

[9] J. Vijande, E. Weissman, A. Valcarce, and N. Barnea, Phys. Rev. D 76, 094027 (2007).

[10] J. -P. Ader, J.-M. Richard, and P. Taxil, Phys. Rev. D 25, 2370 (1982).

[11] S. Zouzou, B. Silvestre-Brac, C. Gignoux, and J.-M. Richard, Z. Phys. C 30, 457 (1986).

[12] C. Semay and B. Silvestre-Brac, Z. Phys. C 61, 271 (1994).

[13] S. Ohkoda, Y. Yamaguchi, S. Yasui, K. Sudoh, and A. Hosaka, Phys. Rev. D 86, 014004 (2012).

[14] G. Bali and M. Hetzenegger, PoS LATTICE2010, 142 (2010).

[15] J. M. Dias, S. Narison, F. S. Navarra, M. Nielsen, and J.-M. Richard, Phys. Lett. B 703, 274 (2011).

[16] T. F. Caramés and A. Valcarce, Phys. Rev. D 90, 014042 (2014).

[17] R. M. Albuquerque, S. H. Lee, and M. Nielsen, Phys. Rev. D 88, 076001 (2013).

[18] J. P. Lees et al., (BABAR Collaboration) Phys. Rev. D 86, 091102(R) (2012).

[19] T. F. Caramés and A. Valcarce, Phys. Rev. D 85, 094017 (2012).

[20] N. Isgur, Phys. Rev. D 60, 054013 (1999).

[21] J. Vijande, A. Valcarce, J. -M. Richard, and P. Sorba Phys. Rev. D 94, 034038 (2016).

[22] M. Oka, K. Shimizu, and K. Yazaki, Phys. Lett. B 130, 365 (1983).

[23] J.-M. Richard, Few Body Syst. 50, 137 (2011). 